\title{
Efecto de los ciclos térmicos de soldadura por arco eléctrico indirecto modificado (AEIM) en las propiedades mecánicas de la aleación AA6061-T6(*)
}

\author{
Ricardo R. Ambriz*, Gerardo Barrera*, Rafael García* y Victor H. López*
}

\begin{abstract}
Resumen
Se presentan los resultados de medición de temperatura durante la soldadura por arco eléctrico indirecto modificado (AEIM) de la aleación AA6061-T6. Éstas, describen los ciclos térmicos de la zona afectada por el calor (ZAC) y en la zona de fusión. Los resultados muestran que las temperaturas máximas medidas en la ZAC se encuentran en un rango de 308 a $693^{\circ} \mathrm{C}$, dependiendo de la posición de los sensores, estas mediciones fueron correlacionadas con los resultados de resistencia a la tracción y la zona de fallo, reportados previamente por los autores ${ }^{[1]}$. Se observó que existe una disminución en la resistencia mecánica de las uniones, debido a los cambios microestructurales por la formación de precipitados sobreenvejecidos, $\beta$ ', de acuerdo con su diagrama de transformación (TTT). Las condiciones de enfriamiento en el baño fundido inherentes a la técnica por AEIM (un solo paso de soldadura), permitieron establecer las características de solidificación y microestructura esperada para una velocidad de enfriamiento determinada.
\end{abstract}

Palabras clave Aleación de aluminio; Baño fundido; Zona afectada por el calor; Transformaciones; Sobreenvejecimiento; Ciclo térmico de soldadura.

\section{Effect of the weld thermal cycles by the modified indirect electric arc (MIEA) on the mechanical properties of the AA6061-T6 alloy}

\begin{abstract}
Results of temperature measurements during welding of $12.7 \mathrm{~mm}$ thick AA6061-T6 alloy plates by modified indirect electric arc (MIEA) are presented. This study describes the thermal cycles of the heat affected zone (HAZ) and also in the fusion zone. Depending upon the position of the transducers, the maximum temperatures measured in the HAZ range from 308 to $693^{\circ} \mathrm{C}$, these measurements were related with the tensile test results, and the failure zone reported previously by the authors [1]. It was observed that, there is a decrease in the mechanical strength of the welded joints, due to the microstructural changes suffered by AA6061-T6 alloy in which formation of the $\beta$ ' occurs according to the TTT transformation diagram. The inherent cooling conditions of the weld pool observed for the MIEA technique (only one pass of welding), have permitted to establish the characteristics of solidification and microstructure for a specific cooling rate.
\end{abstract}

Keywords

Aluminum alloy; Melted pool; Heat affected zone; Transformations; Overaging; Weld thermal cycle.

\section{INTRODUCCIÓN}

La medición de temperatura es un aspecto muy importante en los procesos en los que los efectos térmicos ocasionan cambios que afectan el comportamiento de los materiales, tal es el caso de la soldadura, en donde el calor de aporte debido al arco eléctrico produce cambios microestructurales importantes sobre los materiales soldados y, por lo tanto, en sus propiedades mecánicas ${ }^{[2}$ y 3$]$. Existe una gran variedad de sensores para medir temperatura, los cuales están clasificados en función del rango de medición y tipo de aplicación. Los termopares son los instrumentos de medición de temperatura por excelencia, son elementos termoeléctricos que convierten un cambio de temperatura en un cambio de voltaje (efecto Seebeck); éstos, se fabrican de diversos materiales, por ejemplo, el de Cromel-Alumel (tipo K) que opera en un rango (donde se comporta linealmente), que va desde -270 a $1.260{ }^{\circ} \mathrm{C}\left[{ }^{[4]}\right.$. La medición de temperatura durante el

\footnotetext{
(•) Trabajo recibido el día 30 de enero de 2008 y aceptado en su forma final el día 6 de agosto de 2008.

* Instituto de Investigaciones Metalúrgicas, Universidad Michoacana de San Nicolás de Hidalgo, Apdo. Post 888, Morelia, Mich., México. C.P. 58000. Correo: ricraf74@gmail.com, gbarrera@zeus.umich.mx, rgarcia@zeus.umich.mx.
} 
proceso de soldadura de aleaciones de aluminio tratables térmicamente es de gran interés, debido a la sensibilidad que tienen estas aleaciones a los cambios de temperatura que ocasionan una pérdida de sus estados de endurecimiento, con la consecuente disminución de las propiedades mecánicas ${ }^{[5]}$. Otro punto importante es el agrietamiento por licuefacción que se presenta dentro de una región de alta temperatura en la ZAC ${ }^{[6]}$. Varios autores han estudiado el efecto de la temperatura sobre las propiedades mecánicas y los cambios microestructurales en aleaciones de aluminio tratables térmicamente ${ }^{[3,7 \text { y } 8]}$.

El registro de las variaciones de temperatura durante el proceso de soldadura, tanto en la zona de fusión, la ZAC y en el material base, tiene la finalidad de establecer las transformaciones microestructurales que puede sufrir la aleación y que, inevitablemente, se reflejará en las propiedades mecánicas de la unión soldada. Cabe recordar que el principal mecanismo de endurecimiento en las aleaciones de aluminio tratables térmicamente es la precipitación metaestable de fases endurecedoras, el cual consta de tres etapas: solubilización, temple y envejecimiento (artificial o natural) ${ }^{[9]}$.

El tipo de solidificación de las soldaduras por fusión está totalmente relacionado con el calor de aporte, la composición química del metal de soldadura, la rapidez de crecimiento cristalino, la velocidad de soldeo y el perfil del baño fundido de soldadura. De tal forma, que el tamaño de grano del metal base fundido en el límite de fusión actúa como un substrato para el crecimiento de granos columnares ${ }^{[10]}$. Adicionalmente, la dirección de crecimiento de los granos columnares cambia continuamente a partir de la línea de fusión hacia el centro de la soldadura, debido al cambio correspondiente en la dirección del máximo gradiente de temperatura en el baño fundido. Este, es un problema muy particular de los procesos de alta energía tales como la soldadura por arco sumergido (SAW por sus siglas en inglés) y soldadura de arco eléctrico con material de aporte y gas de protección (GMAW por sus siglas en inglés), donde el crecimiento de grano del metal base es considerable. Más aún, durante soldaduras de pasos múltiples en donde los granos columnares pueden renuclear entre sus límites de un paso de soldadura a otro.

Recientemente, los autores experimentaron con el diseño de junta en la soldadura por arco eléctrico indirecto ${ }^{[11-15]}$ para soldar materiales compuestos de matriz metálica y otros materiales monolíticos. La técnica consiste en posicionar pequeñas placas de alimentación sobre la junta de bordes cuadrados. El arco eléctrico se establece de manera indirecta sobre las placas, formándose un baño líquido de soldadura que, por gravedad y el empuje del arco eléctrico, se alimenta hacia el fondo de la junta, permitiendo soldar espesores de 12,7 mm en una sola pasada de soldadura. Esta técnica, sin embargo, tiene el inconveniente de que las placas de alimentación residuales tienen que ser retiradas. Bajo el mismo esquema, los autores modificaron dicha junta. En lugar de usar las placas de alimentación, se maquinó una pequeña pestaña en la parte superior de las placas a soldar por lo que a este diseño se le denomino arco eléctrico indirecto modificado (AEIM).

El presente trabajo de investigación se llevó a cabo para determinar la relación entre las mediciones de temperatura y el efecto sobre la resistencia mecánica de las uniones, debido al cambio microestructural por la transformación de intermetálicos del tipo $\mathrm{Mg}_{2} \mathrm{Si}$ usando la soldadura por arco eléctrico indirecto modificado. En el mismo contexto, se realizó el estudio de las condiciones y características de enfriamiento del baño de metal líquido, también determinantes en el comportamiento mecánico de una unión soldada. Es importante resaltar que para soldar espesores de 12,7 mm es necesaria la aplicación de varios pasos de soldadura, lo que conduce a un mayor aporte térmico al material base y, como consecuencia, a una mayor afectación térmica y reblandecimiento en la ZAC, de una aleación de aluminio endurecida por precipitación. Así, resulta benéfico el uso de un diseño de junta que permita la unión de estas aleaciones en una sola pasada con la disminución de la perdida de endurecimiento. También, es importante señalar que el mecanizado de la nueva junta no es más complicado ni costoso que la preparación de una junta en simple $\mathrm{V}$.

\section{DESARROLLO EXPERIMENTAL}

\subsection{Variables operativas, condiciones de soldadura y propiedades mecánicas}

El metal base utilizado fue una aleación de aluminio 6061-T6 (Al-Si-Mg) en placa, con un espesor de 12,7 $\mathrm{mm}$. Con el propósito de evaluar las propiedades mecánicas de las juntas soldadas, se utilizaron placas de $70 \mathrm{~mm}$ de ancho por $150 \mathrm{~mm}$ de largo y se mecanizaron probetas de tracción de acuerdo a la norma ASTM B557M-94. Los resultados de estos ensayos fueron presentados en algún trabajo ya citado ${ }^{[1]}$. Con referencia a las zonas donde ocurrió la rotura de las probetas de tracción y las mediciones de microdureza realizadas en la ZAC, se determinó llevar a cabo la medición de ciclos térmicos soldando placas con un ancho de $25 \mathrm{~mm}$ cada una. Además, este ancho permitió colocar los termopares con exactitud. El material de aporte fue un electrodo comercial de alto contenido de silicio (ER4043) con un diámetro de 
1,2 mm. La tabla I muestra la composición química de los materiales empleados, la cuales se obtuvo por espectroscopia de absorción atómica.

Se empleó un proceso de soldadura semiautomático GMAW con argón al 100 \% como gas de protección, con un flujo de 23,6 $1 \mathrm{~min}^{-1}$. Las variables operativas fueron ajustadas para conseguir un modo de transferencia de metal por pulverización, con una corriente aproximada de 230 A, una separación de la boquilla de contacto y material base de $20 \mathrm{~mm}$, polaridad inversa (CDEP), voltaje constante de 23 $V$ y una velocidad de desplazamiento de $3,6 \mathrm{~mm} \mathrm{~s}^{-1}$ de la fuente de calor. En este caso, se utilizó una preparación de junta tipo pestaña, que ha sido denominada arco eléctrico indirecto modificado (AEIM), empleando un precalentamiento de 50, 100 y $150^{\circ} \mathrm{C}^{[1]}$. El diseño de junta y las dimensiones de la misma se ilustran en la figura 1 .

El material base, material de aporte y uniones soldadas, se ensayaron a tracción (empleando un mínimo tres probetas) siguiendo las recomendaciones de la norma ASTM B 557M-94 [16]. La tabla II, presenta los resultados obtenidos para material base y aporte, mientras que los correspondientes a las soldaduras se presentan en el análisis y discusión de resultados. Adicionalmente, se realizaron mediciones de microdureza en el material base y uniones soldadas, aplicando una carga de 0,1 N durante $15 \mathrm{~s}$. El valor promedio de microdureza para el material base fue de $152,5 \mathrm{HV}_{0,01}$, mientras que las uniones soldadas fueron evaluadas mediante barridos de microdureza y correlacionadas con su perfil de soldadura.

\subsection{Medición de temperatura en la ZAC}

Para evitar la interferencia en los patrones de transferencia de calor en el momento de llevar a cabo la soldadura, se elaboraron pequeños termopares, tipo $\mathrm{K}$, con un diámetro de alambre de $0,3 \mathrm{~mm}$. Se fabricaron fundas de plata, con un diámetro exterior e interior de $1,5 \mathrm{~mm}$ y $1 \mathrm{~mm}$, respectivamente, situando los termopares dentro de dichas fundas o aisladores. El aislamiento de los termopares se llevó a cabo rellenando

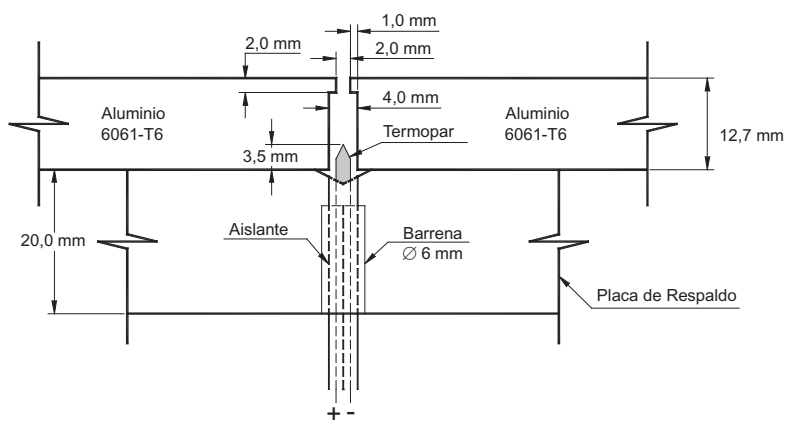

Figura 1. Diseño de junta y ubicación del termopar para medición de temperatura del baño de metal líquido.

Figure 1. Joint design and thermocouple location for measuring the temperature in the liquid metal pool.

Tabla II. Propiedades mecánicas de material base y del material de aporte

Table II. Mechanical properties of the base material and filler wire

\begin{tabular}{lccc}
\hline Aleación & $\begin{array}{c}\text { Resistencia } \\
\text { a Fluencia } \\
\text { (MPa) }\end{array}$ & $\begin{array}{c}\text { Resistencia } \\
\text { a la Tensión } \\
\text { (MPa) }\end{array}$ & $\begin{array}{c}\text { Alargamiento } \\
\text { (\%) }\end{array}$ \\
\hline 6061-T6 & 300 & 328 & 14 \\
ER4043 & 164 & 190 & - \\
\hline
\end{tabular}

el diámetro interior con un cerámico de óxido de silicio $\left(\mathrm{SiO}_{2}\right)$, tal como se presenta en la figura 2 .

La ubicación de los termopares para medición de temperatura en la ZAC, se estableció fijando un sistema de coordenadas rectangular $(\mathrm{X}, \mathrm{Y}, \mathrm{Z})$, cuyo origen se encontraba situado en la cara superior de las chapas de metal base, y en el centro de la preparación de junta (Fig. 3). Se colocaron un total de 10 termopares en las chapas de aleación a soldar. La tabla III resume las posiciones de cada uno de ellos.

Tabla I. Composición química de la aleación 6061 y electrodo ER4043, \% en peso

Table I. Chemical composition of the 6061 alloy and ER4043 filler wire, wt.\%

\begin{tabular}{lccccccccc}
\hline Aleación & $\mathrm{Si}$ & $\mathrm{Fe}$ & $\mathrm{Cu}$ & $\mathrm{Mn}$ & $\mathrm{Mg}$ & $\mathrm{Cr}$ & $\mathrm{Zn}$ & $\mathrm{Ti}$ & $\mathrm{Al}$ \\
\hline $6061-\mathrm{T6}$ & 0,561 & 0,289 & 0,310 & 0,052 & 0,986 & 0,067 & 0,024 & 0,018 & Bal. \\
ER4043 & 5,25 & 0,8 & 0,30 & 0,05 & 0,05 & - & 0,10 & 0,20 & Bal. \\
\hline
\end{tabular}




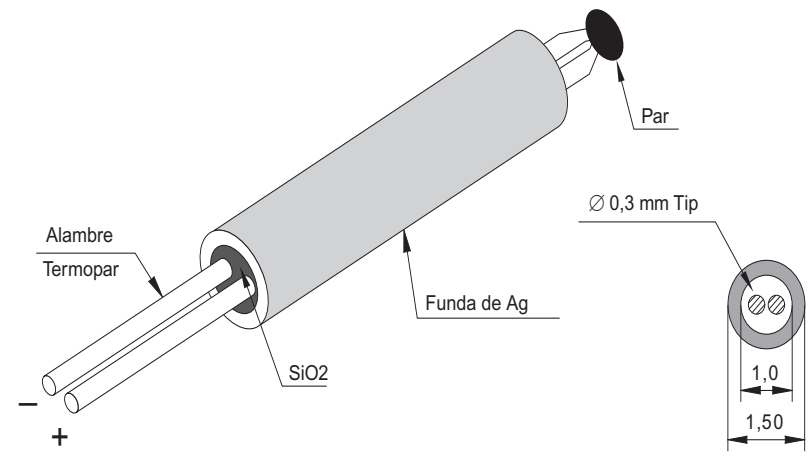

Figura 2. Representación esquemática de termopares tipo $\mathrm{K}$ para medición de la temperatura en la ZAC.

Figure 2. Sketch representation of a $K$ thermocouple for temperature measurement in the HAZ.

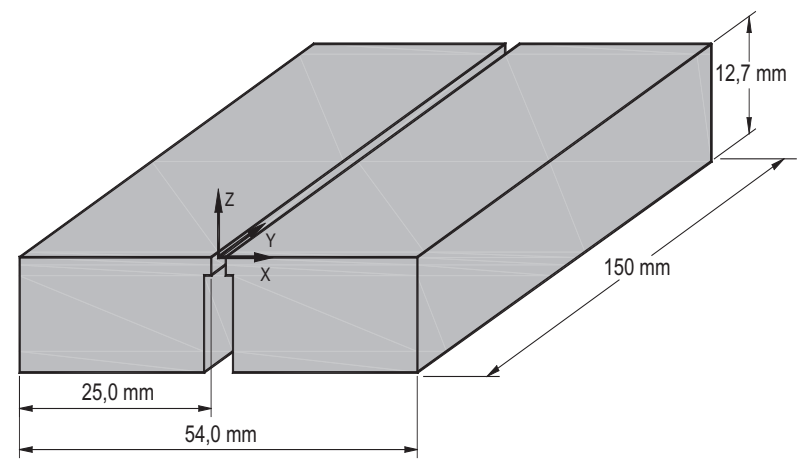

Figura 3. Sistema de coordenadas para la ubicación de termopares.

Figure 3. Coordinate system for the location of the thermocouples.

Tabla III. Posición de termopares

Table III. Location of the thermocouples

\begin{tabular}{ccrr}
\hline Termopar & $\mathrm{X}(\mathrm{mm})$ & $\mathrm{Y}(\mathrm{mm})$ & $\mathrm{Z}(\mathrm{mm})$ \\
\hline 1 & 4,0 & 10,0 & $-9,7$ \\
2 & 6,0 & 42,5 & $-6,7$ \\
3 & 3,0 & 75,0 & $-1,2$ \\
4 & 8,0 & 107,5 & $-10,7$ \\
5 & 10,0 & 140,0 & $-3,7$ \\
6 & $-12,0$ & 10,0 & $-8,7$ \\
7 & $-5,0$ & 42,5 & $-4,7$ \\
8 & $-7,0$ & 75,0 & $-2,7$ \\
9 & $-11,0$ & 107,5 & $-10,7$ \\
10 & $-9,0$ & 140,0 & $-6,7$ \\
Fondo & 0 & 72,0 & $-9,2$ \\
\hline
\end{tabular}

\subsection{Medición de temperatura en el baño fundido de soldadura}

Se colocó un termopar, tipo K, con un diámetro de alambre de 0,8 mm, en el fondo de la preparación de junta a través de un agujero en la placa de respaldo para obtener medidas de temperatura en el baño fundido, su ubicación se ilustra en la figura 1. Es bien conocido que la velocidad de enfriamiento en la zona de fusión es mucho más rápida que la de un lingote $^{[17]}$. Por tanto, es necesario considerar una velocidad de muestreo que permita proporcionar información acerca del fenómeno de solidificación, por lo que las señales se adquirieron a 60 lecturas por segundo; es decir, se hizo la conversión de las señales de analógico a digital a $60 \mathrm{~Hz}$.

\subsection{Digitalización de las señales}

Para el registro de las señales de los termopares se utilizó una tarjeta conectada a una de las ranuras PCI del ordenador, con 16 entradas analógicas diferenciales. Ésta cuenta con una unidad acondicionadora para termopares. Para la fase de adquisición de datos, se utilizaron programas elaborados en lenguaje de programación gráfico $\mathrm{G}$, mejor conocido como "Instrumentación Virtual" (LabVIEW 8.2), en un entorno del sistema operativo Windows XP.

\section{RESULTADOSY DISCUSIÓN}

\subsection{Temperatura en la ZAC y efecto sobre la resistencia mecánica}

La tabla IV muestra las temperaturas máximas medidas para las diferentes condiciones de precalentamiento. Puede observarse que los intervalos de temperatura se incrementan en función de la profundidad a la que se encuentran los sensores con relación a la fuente de calor, así como por las condiciones de precalentamiento empleadas. Por ejemplo, las temperaturas máximas medidas para la posición 1 son de 308,8, 450,8 y 534,1 ${ }^{\circ} \mathrm{C}$. Sin embargo, este comportamiento no se cumple en algunos casos, por ejemplo, para la temperatura máxima medida en la posición 2 con precalentamiento de $100^{\circ} \mathrm{C}$, que tuvo un incremento de $45^{\circ} \mathrm{C}$ con respecto a la medición de temperatura máxima para un precalentamiento de $150^{\circ} \mathrm{C}$. Este fenómeno, que puede explicarse en función del desalineado de la junta con respecto a la fuente de calor y a la naturaleza misma del arco, el cual tiende a mantener su estabilidad hacia un costado, ocasionando que se tenga una mayor 
Tabla IV. Temperaturas máxims medidas en la ZAC

Table IV. Maximum temperatures measured in the $H A Z$

\begin{tabular}{rccc}
\hline Posición & \multicolumn{3}{c}{ Precalentamiento } \\
& $\mathbf{5 0}{ }^{\circ} \mathrm{C}$ & $\mathbf{1 0 0}{ }^{\circ} \mathbf{C}$ & $150{ }^{\circ} \mathbf{C}$ \\
\hline 1 & 308,8 & 450,8 & 534,1 \\
2 & 368,7 & 542,9 & 497,9 \\
3 & 409,5 & 430,3 & 611,8 \\
4 & 469,8 & 462,9 & 577,6 \\
5 & 530,7 & 479,5 & 553,1 \\
6 & 383,1 & 421,3 & 450,2 \\
7 & 461,7 & 384,1 & 577,0 \\
8 & 445,9 & 460,0 & 574,9 \\
9 & Abierto & 456,4 & 614,4 \\
10 & 610,5 & 552,2 & 693,7 \\
\hline
\end{tabular}

cantidad de masa fundida en una de las paredes de la junta, dando lugar a mediciones de temperatura más elevadas. Además, el efecto de conducción de calor debido a la fusión y solidificación de material base y del calor aportado tienen un efecto muy significativo sobre las mediciones de temperatura en los sensores que se encuentran al final de las placas o a diferentes alturas con respecto a la fuente de calor ${ }^{[18]}$.

En la figura 4 se muestran las curvas correspondientes a las mediciones de temperatura dentro de la zona afectada por el calor para la posición 6, correspondiente a $12 \mathrm{~mm}$ de distancia a partir del eje central de la preparación de junta, de acuerdo con la tabla III, para 50,100 y $150^{\circ} \mathrm{C}$ de precalentamiento. Se puede observar que los picos de temperatura máxima alcanzan un valor más elevado conforme se incrementa el precalentamiento. Los valores de las velocidades de calentamiento y enfriamiento calculados se muestran en la tabla $\mathrm{V}$.

Debe notarse como el comportamiento de la velocidad de enfriamiento para cualquiera de las curvas después de transcurrido un determinado tiempo (aproximadamente, 200 s) y, una vez que se ha cruzado a la

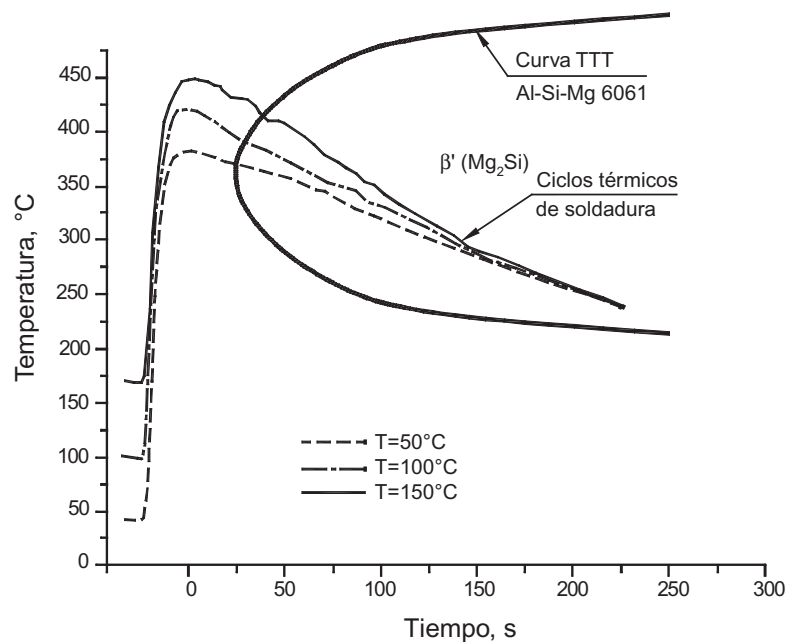

Figura 4. Ciclos térmicos de enfriamiento a 12 $\mathrm{mm}$ de distancia a partir de la fuente de calor y su relación con la curva TTT ${ }^{[5 Y}{ }^{19]}$ de la aleación 6061.

Figure 4. Cooling thermal cycles at $12 \mathrm{~mm}$ from the heat source and its relation with the TTT cur$v e^{[5 Y 19]}$ for 6061 alloy.

curva TTT, es prácticamente la misma. Este comportamiento aporta la información suficiente para explicar porqué la resistencia a la tracción o esfuerzo máximo de las uniones soldadas resulta ser prácticamente la misma, tal y como se puede ver en la tabla VI.

Así mismo, se puede observar la correlación de los ciclos térmicos de enfriamiento con la curva TTT de la aleación, correspondientes al termopar más alejado de la zona de fusión, encontrándose que para cualquier condición de precalentamiento los ciclos de enfriamiento correspondientes rebasan a la curva TTT para la formación de la fase $\beta^{\prime}\left(\mathrm{Mg}_{2} \mathrm{Si}\right)$. También, es de esperar que el crecimiento de precipitados sobreenvejecidos (fase $\beta^{\prime}$ ) sea mayor para la condición de $150^{\circ} \mathrm{C}$ de precalentamiento, favoreciendo para que la zona rotura se encuentre más alejada de la línea de fusión (Fig. 5) ${ }^{[1]}$.

Es evidente que las temperaturas máximas alcanzadas (con relación al termopar 6) no exceden a la

Tabla V. Velocidades de enfriamiento y calentamiento

Table V. Heating and cooling rates

\begin{tabular}{lccc}
\hline & $50{ }^{\circ} \mathrm{C}$ & $100{ }^{\circ} \mathrm{C}$ & $150{ }^{\circ} \mathrm{C}$ \\
\hline Calentamiento, ${ }^{\circ} \mathrm{C} \mathrm{s}^{-1}$ & $16,8\left(1.008{ }^{\circ} \mathrm{C} \mathrm{min}^{-1}\right)$ & $16,5\left(990^{\circ} \mathrm{C} \mathrm{min}^{-1}\right)$ & $8,0\left(480^{\circ} \mathrm{C} \mathrm{min}^{-1}\right)$ \\
Enfriamiento, ${ }^{\circ} \mathrm{C} \mathrm{s}^{-1}$ & $0,6\left(36{ }^{\circ} \mathrm{C} \mathrm{min}-1\right)$ & $0,8\left(48{ }^{\circ} \mathrm{C} \mathrm{min}^{-1}\right)$ & $1,0\left(60^{\circ} \mathrm{C} \mathrm{min}^{-1}\right)$ \\
\hline
\end{tabular}


Tabla VI. Propiedades mecánicas de las juntas soldadas ${ }^{[1]}$

Table VI. Mechanical properties of the welded joints ${ }^{[1]}$

\begin{tabular}{rcccc}
\hline $\begin{array}{c}\text { Precalentamiento } \\
\left({ }^{\circ} \mathrm{C}\right)\end{array}$ & $\begin{array}{c}\text { Alargamiento } \\
(\%)\end{array}$ & $\begin{array}{c}\text { Límite elástico } \\
(\mathrm{MPa})\end{array}$ & $\begin{array}{c}\text { Resistencia tracción } \\
(\mathrm{MPa})\end{array}$ & Zona de fallo \\
\hline 50 & 13,8 & 102,7 & 183,0 & ZAC \\
100 & 15,4 & 101,0 & 181,7 & ZAC \\
150 & 17,2 & 106,5 & 179,3 & ZAC \\
\hline
\end{tabular}

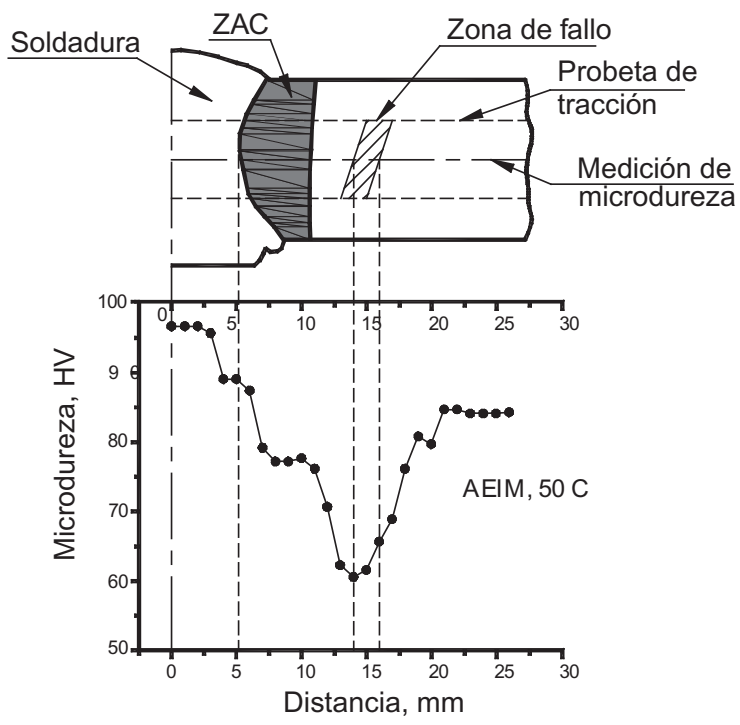

a)

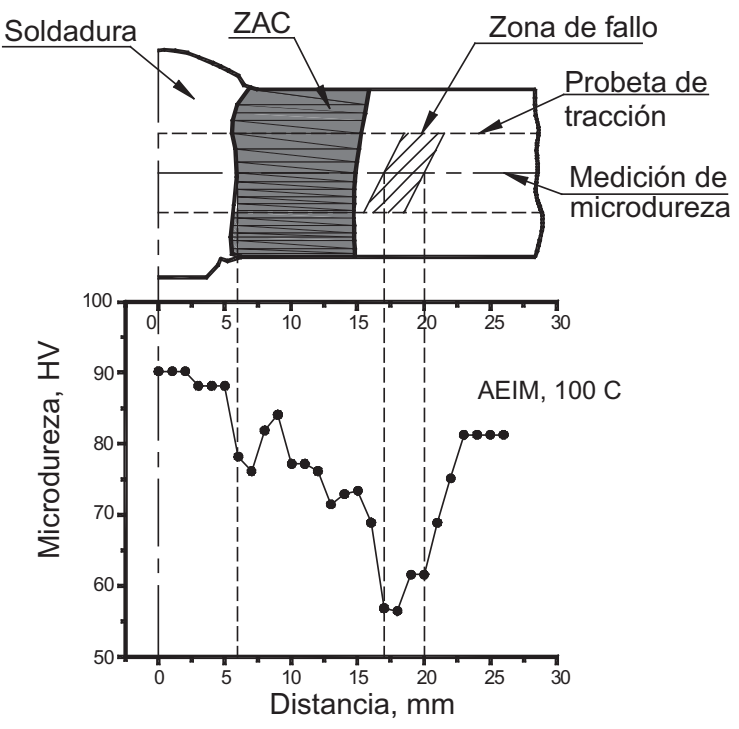

b)

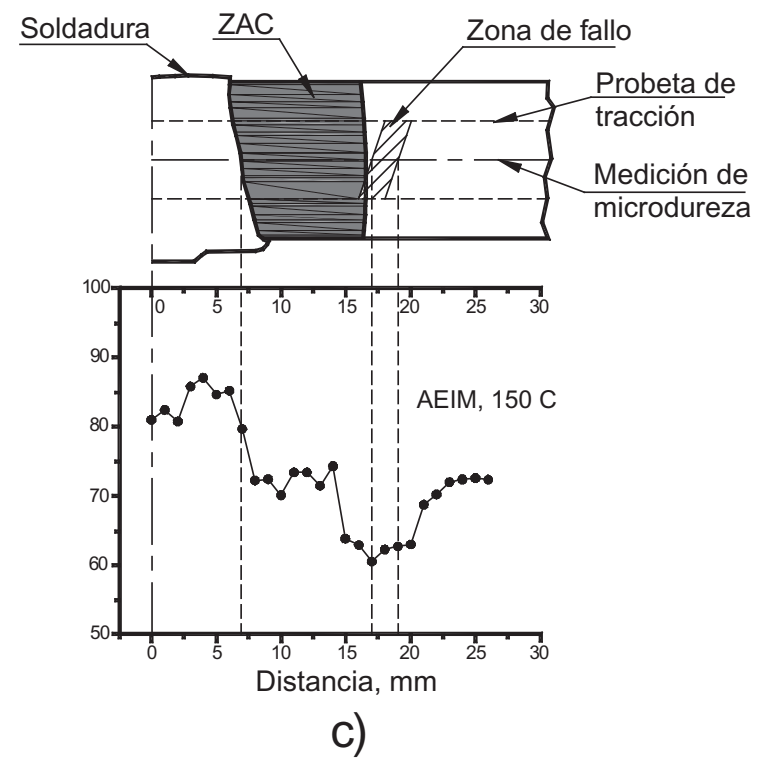

Figura 5. Relación entre los perfiles de microdureza y la zona de fallo, a) AEIM $50^{\circ} \mathrm{C}$, b) AEIM $100{ }^{\circ} \mathrm{C}$ y c) AEIM $150^{\circ} \mathrm{C}$.

Figure 5. Relation between the microhardness profiles and the failure zone, a) MIEA $50{ }^{\circ} \mathrm{C}$, b) MIEA $100{ }^{\circ} \mathrm{C}$ y c) MIEA $150{ }^{\circ} \mathrm{C}$. 
temperatura de solubilización de la aleación 6061, la cual se encuentra alrededor de los $530^{\circ} \mathrm{C}$. Sin embargo, éstas temperaturas se encuentran por encima de la temperatura de formación de zonas de Guinier-Preston, de acuerdo con la secuencia de precipitación para las aleaciones de $\mathrm{Al}-\mathrm{Si}-\mathrm{Mg}{ }^{[20]}$. Las temperaturas máximas alcanzadas son de 383,421 y $450^{\circ} \mathrm{C}$ para sus respectivos precalentamientos, que son muy aproximadas a los valores correspondientes reportados por O.R. Myhr ${ }^{[7]}$, quien establece que la transformación de precipitados $\beta$ " a $\beta$ ' ocurre cuando la temperatura pico rebasa los $315^{\circ} \mathrm{C}$, con un intervalo de tiempo de por lo menos $10 \mathrm{~s}$ (condición que se cumple en el caso de las curvas de la figura 4 ) y a $390^{\circ} \mathrm{C}$, la fase $\beta$ ' comienza a ser el constituyente microestructural dominante. Lo anterior también tiene correspondencia con las investigaciones realizadas por V. Malin ${ }^{[2]}$ acerca de la relación que existe entre la medición de temperatura máxima medida en la zona afectada por el calor y la localización del fallo en aleaciones de aluminio 6061 T6 extruidas y soldadas de forma convencional por medio de preparación de junta en simple $\mathrm{V}$, encontrando que el valor mínimo de microdureza y localización de la fractura está asociada a picos de temperatura de alrededor de $380^{\circ} \mathrm{C}$.

Por otra parte, la fase $\beta$ " con morfología acicular es la que produce el mayor efecto sobre el endurecimiento de la aleación 6061-T6, según lo establecen Dutta y Allen ${ }^{[20]}$, mientras que la fase $\beta$ ', que tiene una forma de barra, crecerá dependiendo del incremento de temperatura en la ZAC originando el sobreenvejecimiento y, por tanto, su incoherencia con la matriz de aluminio. Lo anterior, se comprueba con la imagen de la figura 6 , correspondiente a la fractura de una de las uniones por AEIM, en donde se observa una partícula de $\mathrm{Mg}_{2} \mathrm{Si}$ de gran tamaño, entre otras.

Es evidente que la fractura ha sido ocasionada por el reblandecimiento en la ZAC por la formación de partículas de precipitado $\beta$ ' y/o tipo $\beta$ (de equilibrio), producto de la inestabilidad termodinámica de los precipitados $\beta$ " durante un proceso de soldadura por fusión. Sin embargo las propiedades mecánicas obtenidas después del proceso de unión por la técnica de AEIM (con una sola pasada de soldadura), son considerablemente mayores en relación a soldaduras realizadas de forma convencional, con una preparación de junta en simple V y 3 ó 4 pasos de soldadura ${ }^{[1,21,22]}$.

\subsection{Temperatura en el baño fundido de soldadura}

La figura 7 presenta el grafico de los datos obtenidos por el sistema de adquisición de datos para la medición de temperatura en el baño fundido de soldadu-

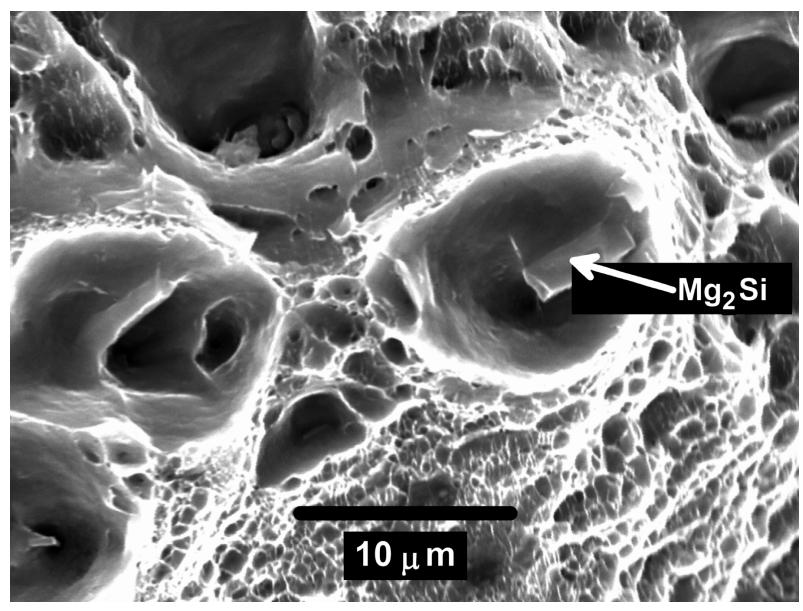

Figura 6. Fractura de una soldadura 6061-T6, mostrando partículas del tipo $\mathrm{Mg}_{2} \mathrm{Si}$.

Figure 6. Fracture of the 6061-T6 weld showing $\mathrm{Mg}_{2}$ Si particles.

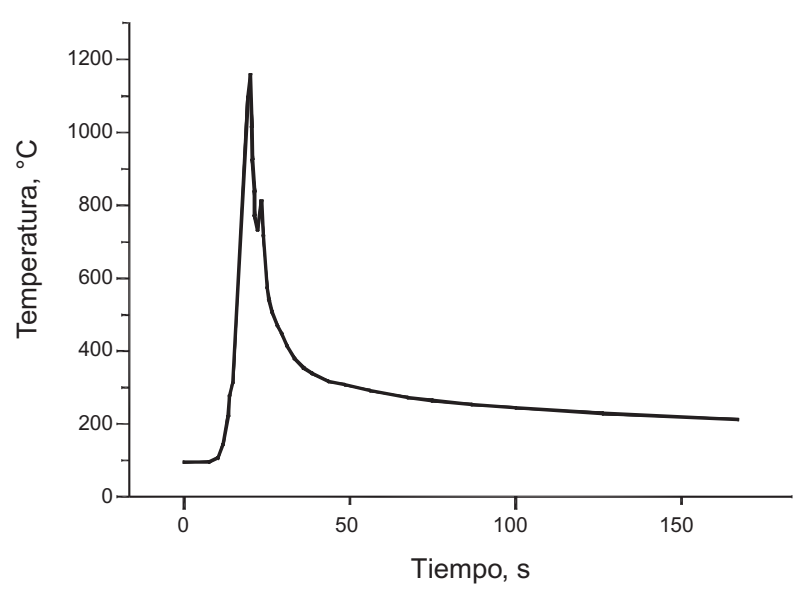

Figura 7. Grafico de medición de temperatura en el metal líquido de soldadura.

\section{Figure 7. Profile of the temperature measured in the weld pool.}

ra, de acuerdo con la posición del termopar de la figura 1, en la cual se puede observar que el proceso de adquisición de datos comenzó cuando la temperatura mínima era de $97^{\circ} \mathrm{C}$ (tiempo de 0 s) y se alcanzó una temperatura máxima de $1.157^{\circ} \mathrm{C}$, correspondiente a 1.198 lecturas $(19,96 \mathrm{~s})$, por lo cual se obtuvo un incremento de temperatura de $1.060^{\circ} \mathrm{C}$.

Realizando un análisis de los datos obtenidos, se observó que, para un total de 615 lecturas (10,25 s), es decir cuando $\mathrm{T}=111^{\circ} \mathrm{C}$, el incremento de la velocidad de calentamiento fue insignificante, a razón de $1,38^{\circ} \mathrm{C} \mathrm{s}^{-1}$. Este incremento corresponde a la 
porción recta del inicio de la curva de la figura 7, por lo cual es posible tomar como tiempo de inicio de medición de temperatura al valor de 615 lecturas, aspecto que resulta evidente debido a que la pendiente de la curva a partir de este valor comienza a ser muy prolongada. Con base en lo anterior se puede determinar que el tiempo requerido para alcanzar la temperatura máxima es de $9,7 \mathrm{~s}$, pudiéndose calcular la velocidad de calentamiento a través de la pendiente de la curva de la figura 7, por medio de la siguiente relación:

$$
G_{t}=\frac{T_{p}-T_{i}}{t}=\frac{(1157-111)^{\circ} \mathrm{C}}{9,7 s}=107,8^{\circ} \mathrm{Cs}^{-1}
$$

donde, $G_{t}$ es la velocidad de calentamiento o enfriamiento, $T_{p}$ es la temperatura máxima y $T_{i}$ es la temperatura inicial registrada por el termopar.

La distancia real del termopar con respecto al inicio del ciclo térmico de la soldadura (inicio del arco) fue de $72 \mathrm{~mm}$ (Tabla III), lo que teóricamente debe corresponder al tiempo en donde la temperatura es máxima, es decir 19,96 s. Lo anterior puede comprobarse en función de la velocidad de soldeo empleada por el proceso de soldadura $\left(3,6 \mathrm{~mm} \mathrm{~s}^{-1}\right)$ y haciendo uso de la definición para determinar la velocidad instantánea, se puede calcular la distancia de la siguiente manera:

$$
\mathrm{d}=\mathrm{vt}=(3,6 \mathrm{~mm} / \mathrm{s})(19,96 \mathrm{~s})=71,85 \mathrm{~mm}
$$

El valor calculado resulta ser una distancia que corresponde, aproximadamente, con la distancia a la cual se encuentra ubicado el termopar. Por lo tanto, existe la evidencia suficiente para determinar que el material de aporte es suministrado por efecto de la fuerza de gravedad hacia la junta de las placas a unir y no por medio de un posible arrastre de metal fundido por delante de la fuente de calor (es decir la fuente de calor es perpendicular al sensor de temperatura). Un punto importante a tomar en consideración es que la temperatura máxima medida en el metal de soldadura, es la temperatura correspondiente a una distancia de $3,5 \mathrm{~mm}$ por encima de la placa de respaldo y no es la temperatura a la cual se desprenden las gotas de material de aporte por el proceso de soldadura $^{[8]}$.

La figura 8 es la grafica correspondiente al intervalo de tiempo en donde la temperatura es máxima en el metal líquido de soldadura. Realizando un análisis en función de la temperatura de fusión del material de aporte $\left(630^{\circ} \mathrm{C}\right)$ y su temperatura eutéctica $\left(581^{\circ} \mathrm{C}\right)$, es posible determinar el tiempo de enfriamiento para un descenso de temperatura de $49^{\circ} \mathrm{C}$, correspondiente a $0,76 \mathrm{~s}$, por lo tanto la velocidad de enfriamiento será:

$$
\mathrm{G}_{\mathrm{t}}=\frac{49^{\circ} \mathrm{C}}{0,76 \mathrm{~s}}=64,47^{\circ} \mathrm{Cs}^{-1}
$$

Esta velocidad de enfriamiento se encuentra por debajo de los valores altos para un proceso GMAW[23]. Sin embargo, el grado de subenfriamiento previo experimentado en el proceso de solidificación, favorece la obtención de un tamaño de grano fino, tal y como se muestra en la micrografía de la figura 9, correspondiente a la porción central de la soldadura para una unión por AEIM precalentada a $150^{\circ} \mathrm{C}$.

Una vez alcanzada la temperatura máxima, está comienza a disminuir debido al desplazamiento de la fuente de calor en función de la velocidad de soldeo del proceso de soldadura, según lo establecido en las investigaciones para las fuentes de calor en movimiento por Rosenthal ${ }^{[18]}$. Durante el enfriamiento, se comienza a suministrar energía a las paredes laterales de la preparación de la junta, experimentándose un descenso en la temperatura hasta llegar a un mínimo correspondiente a $737^{\circ} \mathrm{C}$, que se encuentra por encima del punto de fusión del material base $(652$ ${ }^{\circ} \mathrm{C}$ ) y del material de aporte $\left(630^{\circ} \mathrm{C}\right)$. En este punto, se funden, totalmente, las paredes laterales, por lo que existe un desprendimiento de calor latente de fusión que representa a la energía generada durante la transformación líquido-sólido, el cual es absorbido por el metal líquido aumentándose su temperatura

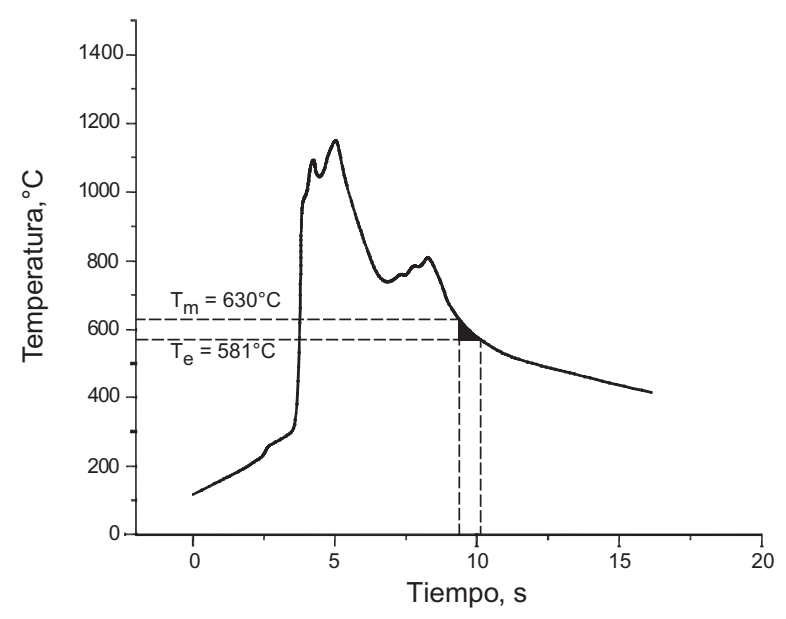

Figura 8. Perfil de temperatura del metal de soldadura donde la temperatura es máxima y ocurre la transformación líquido-sólido.

Figure 8. Temperature profile of the weld pool in which the temperature is maximum and the liquid-solid transformation takes place. 


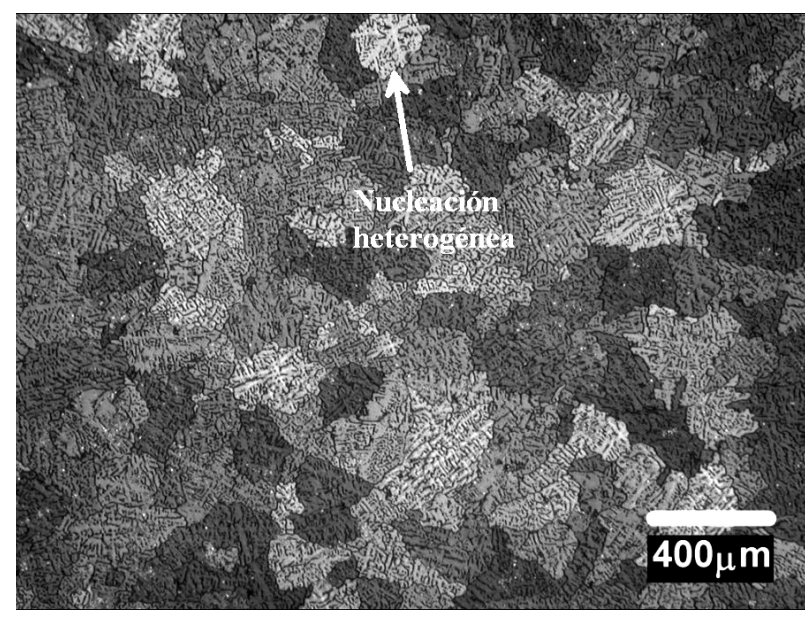

Figura 9. Microestructura del metal de la soldadura de una junta precalentada a $150{ }^{\circ} \mathrm{C}$, mostrando nucleación heterogénea.

Figure 9. Microstructure of the weld metal in a joint preheated to $150^{\circ} \mathrm{C}$, showing heterogeneous nucleation.

alrededor de $75^{\circ} \mathrm{C}$ en un corto tiempo, de $1,41 \mathrm{~s}$, llegando a un máximo de $812^{\circ} \mathrm{C}$.

La teoría clásica para la nucleación heterogénea explica que la forma en la que se disipa el calor latente de fusión determina el mecanismo de crecimiento y la estructura final del material solidificado. Esta teoría fue la base para el desarrollo de la idea de García et. al. ${ }^{[13]}$ quienes explicaron, esquemáticamente, el comportamiento de la velocidad de enfriamiento en los procesos de soldadura tradicional y por arco eléctrico indirecto (AEI), (Fig. 10).

Se puede observar que en el caso de la solidificación para la soldadura tradicional, posterior al subenfriamiento, existe una estabilización térmica (crecimiento columnar) y, después, si las condiciones se presentan, puede darse crecimiento de grano equiaxial debido al subenfriamiento constitucional. Sin embargo, en el caso de la soldadura por arco eléctrico indirecto se presenta un subenfriamiento del líquido, posteriormente, una recalescencia y, finalmente, un enfriamiento continuo a una velocidad de enfriamiento alta.

Por lo tanto, es importante evaluar la variación de la temperatura del baño fundido de soldadura $G_{L}$ con respecto a la distancia de la fuente de calor, correspondiente al límite de fusión (sólido-líquido), definido por:

$$
G_{L}=\frac{d T_{L}}{d x}
$$

Partiendo del grafico de medición de temperatura en el baño de fusión, se puede obtener el segmen-

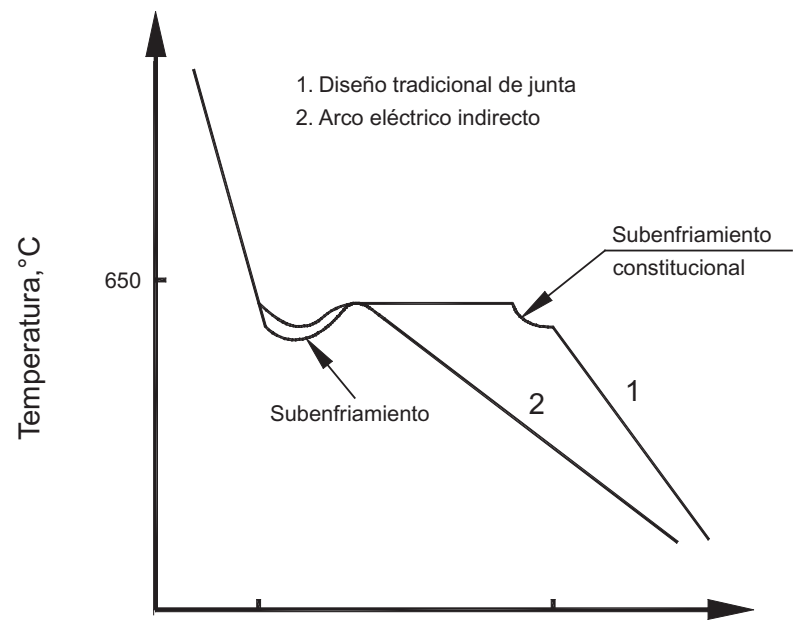

Tiempo, s

Figura 10. Comparación de los patrones de enfriamiento entre soldadura tradicional y arco eléctrico indirecto[ ${ }^{[13]}$.

Figure 10. Comparison of cooling patterns between traditional and indirect electric arc welding ${ }^{[13]}$.

to de curva correspondiente al enfriamiento del metal de soldadura líquido (Fig. 11).

Se puede observar que la transformación de líquido a sólido, durante el proceso de solidificación, se presenta de forma continua desde la temperatura de fusión de la aleación del material de aporte $\left(630^{\circ} \mathrm{C}\right)$ y hasta la de solidificación del mismo $\left(581^{\circ} \mathrm{C}\right)$.

Partiendo de la aproximación polinomial obtenida en el grafico de la figura 11 se puede realizar el

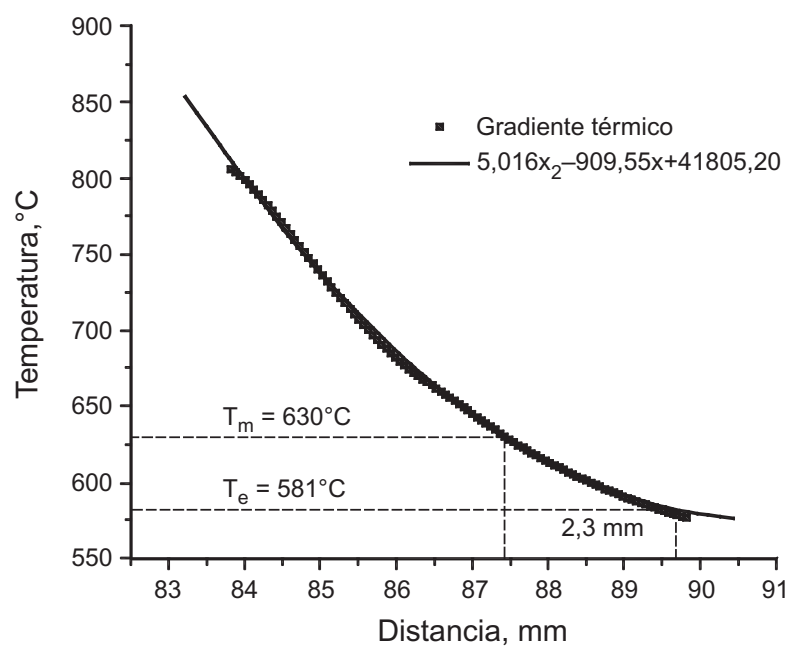

Figura 11. Porción de la curva de enfriamiento en el metal líquido de soldadura.

Figure 11. Fraction of the cooling curve of the weld pool. 
cálculo correspondiente a la variación de la temperatura en el centro del baño fundido, en relación al avance de la fuente de calor, de la siguiente manera:

$$
G_{L}=\frac{d}{d x}\left(41805,2-909,55 x+5,016 x^{2}\right)=-909,55+10,03 x
$$

De la misma figura 11 , se obtiene la distancia recorrida por la fuente de calor desde una temperatura de 811,9 a $581^{\circ} \mathrm{C}$, la cual es de $6 \mathrm{~mm}$. Por lo tanto, se genera un gradiente de temperatura de $849,37^{\circ} \mathrm{C}$ $\mathrm{mm}^{-1}$. De acuerdo con el diagrama de variación microestructural desarrollado por Kurtz et al. ${ }^{[24]}$, el tipo de microestructura el centro de la soldadura corresponde un tipo celular dendrítico (Fig. 9).

\section{CONCLUSIONES}

- La velocidad de enfriamiento a cualquier condición de soldadura en AEIM, rebasa la curva TTT de formación de precipitados, $\beta$ ', y, a un tiempo determinado, estas velocidades tienden a igualarse, por lo que la resistencia mecánica prácticamente es la misma debido a la transición de precipitados de la fase $\beta$ " a $\beta$, .

- La incoherencia de la fase $\beta^{\prime}$ con la matriz disminuye el grado de endurecimiento dentro de la ZAC y la zona de ruptura, después del ensayo de tracción, depende del precalentamiento, en soldaduras por AEIM.

- La medición de temperatura en el baño fundido, permitió determinar las características de enfriamiento en el metal líquido y calcular, de forma aproximada, el tipo de microestructura esperado en el metal de soldadura posterior a la solidificación.

\section{Agradecimientos}

Los autores desean agradecer al Consejo Nacional de Ciencia y Tecnología por su apoyo, a la Universidad Michoacana de San Nicolás de Hidalgo y en especial al Instituto Tecnológico de Morelia por todas las facilidades prestadas para el uso de sus laboratorios.

\section{REFERENCIAS}

[1] R. R. Ambriz, G. Barrera y R. García, SEI 11 (2006) 10-17.

[2] V. Malin, Weld. J. 74 (1995) 305s-318s.

[3] Y. Li, L. E. Murr y J. C. Mc Clure, Mat. Sci. Eng. A 271 (1999) 213-223.

[4] N. A. Anderson, Instrumentation for Process Measurement and Control., pp. 131-133.

[5] ASM, Heat Treater's Guide: Nonferrous Alloys, p. 204.

[6] C. Huang y S. Kou, Weld. J. (2004) 111s-122s.

[7] O. R Myhr, O. Grong, H. G. Fjaer y C. D. Marioara, Acta Mater. 52 (2004) 4.997-5.008.

[8] M. J. Lu y S. Kou, Weld. J. (1989) 382-s-388-s.

[9] J. E. Hatch, Aluminum Properties and Physical Metallurgy, Ohio, 1993, pp. 134-148.

[10] G. J. Davies y J. G. Garland, Int. Metall. Rev. 20 (1975) 83-106.

[11] R. Garcia, V. H. Lopez, J. Mater. Sci. 42 (2006) 7856-7963.

[12] R. Garcia, V. H. Lopez, A. R. Kennedy y G. Arias, J. Mater. Sci. 42 (2007) 7794-7800.

[13] R. Garcia, V. H. Lopez, E. Bedolla y A. Manzano, J. Mater. Sci. Lett. 21 (2002) 1.965-1.967.

[14] R. Garcia, V. H. Lopez, E. Bedolla y A. Manzano, J. Mat. Sci. 38 (2003) 2.771-2.779.

[15] V. H. López. R. García y E. Bedolla, Metall. Mater. Trans. B (2002) 932-937.

[16] ASTM, Standard Test Methods of Tension Testing Wrought and Cast Aluminum-and MagnesiumAlloy Products [Metric], ASTM, 1994, pp. 419. 429.

[17] O. O. Grong, Metallurgical Modelling of Welding, The Institute of Materials, 1997, pp. 221-278.

[18] D. Rosenthal, Trans. ASME (1946) 849-866.

[19] T. Sheppard, Mater. Sci. Tech. 4 (1988) 636.

[20] I. Dutta y S. M. Allen, J. Mater. Sci. Lett. 10 (1991) 323-326.

[21] A. O. Kluken y B. Bjorneklett, Weld. J. (1997) 39-44.

[22] L. A. Guiterrez, G. Neye y E. Zschech, Weld. J. (1996) 116s-121s.

[23] S. A. David y J. M. Vitek, Mat. Rev. 34 (1989) 213-245.

[24] W. Kurz y D. J. Fisher, Fundamentals of Solidification, Aedermannsdorf (Switzerland), 1989. 